\title{
Kinetics of Hydrolyzing Isolated Soy Protein by an Endopeptidase and its Conceptual Application in Process Engineering
}

\author{
Zebin Wang* ${ }^{*}$ Jason Lombardi, Jessica Shaffer, and Ted Wong \\ Discovery Research, Solae, LLC, 4300 Duncan Ave, St Louis, MO, USA 6311 \\ zwang@solae.com
}

Received: 12 April 2011; Published online: 18 April 2012

\begin{abstract}
A response study and the effects of different parameters ( $\mathrm{pH}$, temperature and enzyme dose) on kinetics of isolated soy protein hydrolysis by a trypsin-like endopeptidase (TL1) were conducted. Degree of hydrolysis $(\% \mathrm{DH})$ data varied at different times under different hydrolysis conditions. Fitting the kinetics data to Michaelis-Menten kinetics model did not result in reasonable kinetic parameters, which implied that Michaelis-Menten kinetics was invalid for such a hydrolysis process. A kinetics model proposed by (Gonzalez-Tello, Camacho, Jurado, Paez, \& Guadix, 1994) was found to fit the kinetics curve well and resulted in acceptable model parameters. A simple simulation example was performed to demonstrate the concept of how the kinetics equation could be applied in process engineering.
\end{abstract}

Keywords: isolated soy protein; protein hydrolysis; hydrolysis kinetics; Trypsin-like endopeotidase; process simulation

\section{Introduction}

Soy protein is a high quality protein with more health benefits than animal proteins. The Food \& Drug Administration (FDA) approved a health claim on soy protein stating (Food and Drug Administration, 1999)

"25 grams of soy protein a day, as part of a diet low in saturated fat and cholesterol, may reduce the risk of heart disease"

To extend its application in food products, enzymatic hydrolysis is extensively used to improve the functional properties of soy protein, including solubility, emulsification and foaming characteristics (Pusky, 1975; Were, Hettiarachchy, \& Kalapathy, 1997). Hydrolysis may also create bioactive peptides for additional health benefits
(Gibbs, Zougman, Masse, \& Mulligan, 2004; Erdmann, Cheung, \& Schröder, 2008). In an industrial hydrolysis facility, it is difficult to control the conditions used during reaction $(\mathrm{pH}$, temperature, enzyme concentration, etc.) to be as consistent as laboratory bench experiments. A wide range of operating parameters will result in high product variation, while a narrow range requires higher operation cost. Experimentally determining such control limits is expensive due to the huge trial cost. Therefore, process modeling and simulation is valuable to narrow down the conditions required for process scale up as well as operating control specifications. Commercial Finite Element Analysis ( $F E A$ ) software is capable of performing such kinds of modelling and simulation. However, the accuracy of simulation strongly depends on the data input. Enzymatic kinetics is one of the most critical inputs. 
Hydrolysis Kinetics of Soy Protein and its Application | 27

Classical enzymatic kinetics is expressed by the Michaelis-Menten model, which describes the ideal enzymatic reaction. Ideal enzymatic reactions are characterized by homogeneity of enzyme and substrate, no enzyme inactivation during reaction, and the substrate being the only inhibiting factor. Like other food proteins, commercial soy proteins are not able to form true solutions, therefore the access of enzyme to cleaving sites may be limited (Markovic, Topolovec, Maric, \& Johanides, 1988). Furthermore, enzymes may lose activity during hydrolysis (Gonzalez-Tello et al., 1994). Due to these complexities, it is difficult to describe such a hydrolysis process using a simple kinetics model (Markovic et al., 1988). Several authors have proposed kinetics models for actual hydrolysis systems (Gonzalez-Tello et al., 1994; O'Meara \& Munro, 1985; Ely, Williamson, Guenther, Hyman, \& Arp, 1995; Barros \& Malcata, 2002). These models are based on different assumptions and show good fit to experimental data. Trypsinlike endopeptidase (TL1) is used to create soy protein hydrolysate with improved flavor (U.S. Patent No. 305,212, 2008). In the present work, isolated soy protein was hydrolyzed by TL1 under different conditions. Degree of hydrolysis $(\% \mathrm{DH})$ was measured for samples obtained at different reaction times. Kinetics data were fitted to the Michaelis-Menten model and the model proposed by (Gonzalez-Tello et al., 1994). A simple example of hydrolysis under random temperature within a given range was simulated to demonstrate that the kinetics equation generated can be used to simulate industrial manufacture process.

\section{Experimental}

\subsection{Sample Procedure}

Isolated soy protein ( Supro @ 760 , Solae, LLC ) was dispersed in tap water at $14 \%$ concentration (w:w). Hydrolysis was performed in a beaker with overhead stirring in water bath at desired temperature; $\mathrm{pH}$ was adjusted to desired value by a $\mathrm{pH}$-titrator ( DL50, Mettler Toledo ) with $1 \mathrm{~N} \mathrm{NaOH}$. Hydrolysis was started by adding desired amount of Trypsin-like endopep- tidase (TL1, Novozymes). Different sets of $\mathrm{pH}$, temperature and enzyme dose were applied for hydrolysis following a surface response design using MiniTab 15. Under each set of hydrolysis conditions, samples were removed from the beaker at $0,5,10,20,40,60$ and 90 minutes, followed by enzyme deactivation in boiling water for $5 \mathrm{~min}$, and then stored at $-20^{\circ} \mathrm{C}$ for analysis.

\subsection{Degree of Hydrolysis}

Degree of hydrolysis (\%DH) was measured using o-phthaldialdehyde (OPA) method as described by Gonzalez-Tello et al. (1994).

\section{Results and Discussion}

\subsection{Model description}

\section{Applying Michaelis-Menten model to the hydrolysis process}

The Michaelis-Menten kinetics model describes the reaction as:

$$
\frac{d P}{d t}=\frac{V_{m} S}{K_{m} S}
$$

in which $P$ and $S$ are product and substrate concentration, respectively. For a protein hydrolysis reaction, the product is reflected by an increase in free $\mathrm{NH}_{2}$ or $\mathrm{COOH}$, which is characterized by degree of hydrolysis (\% DH). Substrate is expressed as the peptide bonds in the protein which are able to cleave. Based on such consideration, left side of equation 1 can be expressed as:

$$
\frac{d P}{d t}=M \frac{d D H}{d t}
$$

in which, $M$ is the total peptide bond of unit mole protein. Peptide bond able to be cleaved is a fraction of total peptide bond. Therefore,

$$
S_{0}=f M
$$

in which, $f$ is the fraction of total peptide bond that can be cleaved. During hydrolysis,

$$
S=S_{0}-D H \cdot M
$$


Plug equations 2- 4 into equation 1 ,

$$
\frac{d D H}{d t}=\frac{V_{m}(f-D H)}{K_{m}+M(f-D H)}
$$

Define

$$
V_{m}^{*}=\frac{V_{m}}{M} K_{m}^{*}=\frac{K_{m}}{M}
$$

Equation 5 can be re-organized to:

$$
\left(\frac{K_{m}^{*}}{V_{m}(f-D H)}+\frac{1}{V_{m}^{*}}\right) d(f-D H)=-d t
$$

With the initial condition, that is:

$$
D H=0 \text { at } t=0
$$

Equation 7 can be solved and organized as:

$$
t=\frac{K_{m}^{*}}{V_{m}^{*}} \ln \frac{f}{f-D H}+\frac{D H}{V_{m}^{*}}
$$

Fitting the experimental data of $\mathrm{DH}$ as function of time to equation 9 will provide the model parameters.

\section{Gonzalez-Tello's model}

Gonzalez-Tello et al. (1994) derived a kinetics equation for protein hydrolysis based on the assumption of: (i) the hydrolysis reaction is zeroorder for the substrate; (ii) the enzyme denatures simultaneously via a second-order kinetic process due to free enzyme attacking enzyme bound to the substrate. The differential equation is:

$$
\frac{d D H}{d t}=a e^{-b \cdot D H}
$$

in which a and b are model parameters. And the integral form is:

$$
D H=\frac{1}{b} \ln (1+a \cdot b \cdot t)
$$

\subsection{Fitting kinetics curves to Michaelis-Menten model}

TL1 has high specificity for hydrolysis of soy protein at a peptide with arginine or lysine (U.S. Patent No. 305,212, 2008). The total fraction of arginine and lysine is around $14 \%$, which was considered as the theoretical maximum $\% \mathrm{DH}$ for soy protein hydrolyzed by TL1. In equation 9 , this maximum of $\% \mathrm{DH}$ is the constant $f$. Figure 1 shows two typical kinetics curves fitted to equation 9. Apparently the fittings are reasonable, but the parameters $K_{m}$ and $V_{m}$ are negative, which have no physical meanings. If the non-negative constraints were applied in the fitting, the data were not able to be fitted to the equation successfully. In addition, plot of $\mathrm{DH} \%$ versus time with arbitrary value of $K_{m}$ and $V_{m}$ showed that $\% \mathrm{DH}$ linearly increased with time before $\% \mathrm{DH}$ reached $8 \%$ or so (plot not shown). However, among all the experimental data, $\% \mathrm{DH}$ reached plateau far below $8 \%$. Fitting other set of kinetics curves to Michelis-Menten model resulted in similar observations, suggesting that the Michaelis-Menten model was invalid for this hydrolysis reaction.

Table 1: Model parameters (a and b) for Gonzalez-Tello's model (Gonzalez-Tello et al., 1994)

\begin{tabular}{cccccc}
\hline Set & $\mathrm{pH}$ & $\begin{array}{c}\mathrm{T} \\
{\left[{ }^{\circ} \mathrm{C}\right]}\end{array}$ & $\begin{array}{c}\text { Dose } \\
{[\mathrm{mg} / \mathrm{kg}]}\end{array}$ & $\mathrm{a}^{*}$ & $\mathrm{~b}^{*}$ \\
\hline 1 & 7.5 & 50 & 150 & 0.5941 & 0.866 \\
2 & 8 & 55 & 100 & 0.1601 & 1.7338 \\
3 & 8.5 & 60 & 150 & - & - \\
4 & 8.5 & 50 & 50 & 0.02775 & 0.8635 \\
5 & 7.5 & 60 & 50 & 0.2515 & 2.8255 \\
6 & 7.5 & 50 & 50 & 0.06289 & 1.3 \\
7 & 8.5 & 60 & 50 & - & - \\
8 & 7.5 & 60 & 150 & - & - \\
9 & 8.5 & 50 & 150 & 0.1438 & 0.6403 \\
10 & 8 & 63.2 & 100 & 0.1598 & 5.7341 \\
11 & 7.2 & 55 & 100 & 0.4272 & 1.1641 \\
12 & 8 & 55 & 182 & 0.5845 & 1.093 \\
13 & 8.8 & 55 & 100 & 0.09367 & 2.0621 \\
14 & 8 & 46.8 & 100 & 0.07663 & 0.6407 \\
15 & 8 & 55 & 18.35 & 0.04965 & 2.9388 \\
16 & 8 & 55 & 100 & 0.4378 & 1.3132 \\
\hline
\end{tabular}

* no reliable a, b values were obtained by fitting experimental data to model

\section{Fitting kinetics curves to Gonzalez-Tello's model (Gonzalez-Tello et al., 1994)}

Kinetics curves obtained under several different conditions were fitted to Gonzalez-Tello's model 


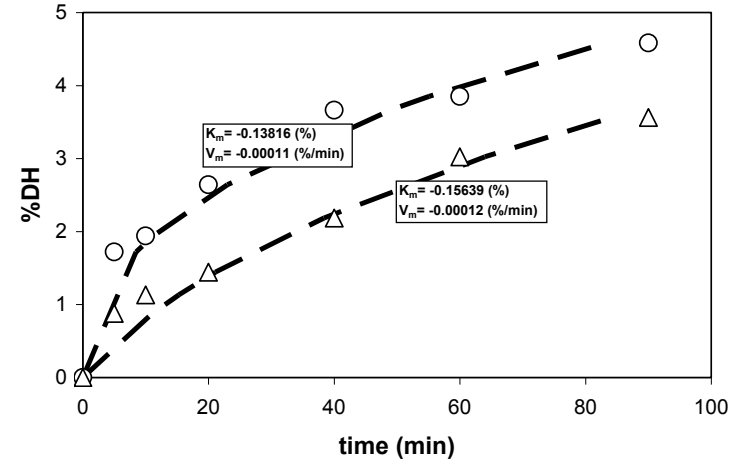

Figure 1: Fitting two typical kinetics curves to Michaelis-Menten model through equation 9, $\mathrm{f}$ value of 0.14 was applied. The circles (D) and triangles $(\mathrm{F})$ are for kinetics curve at $\mathrm{pH} 7.5$ and $\mathrm{pH} 8.5$ respectively, temperature and enzyme dose for both are $50{ }^{\circ} \mathrm{C}$ and $150 \mathrm{mg} / \mathrm{kg}$ protein. Dashed lines (-) are fitted curves, and the fitted model parameters are listed in the box on the curves.

(figure 2). The data fitted the model fairly well. A surface response design experiment for $\mathrm{pH}$, temperature and enzyme dose was run. Model parameters for different hydrolysis conditions are listed in table 3.2, among which, hydrolysis conducted under condition sets 3,7 and 8 was poor, resulting in DH lower or around $1 \%$. The kinetics trend was not demonstrated due to the variation of $\% \mathrm{DH}$ comparable to the true value. Consequently, no reasonable values of $a$ and $b$ were obtained for these condition sets.

\section{Physical meaning of the model parameters}

The left side of equation 10 is the increasing rate of $D H$. At time zero, $D H$ is zero, therefore the right side of equation 10 is reduced to constant $a$. Thus, the physical meaning of a is the initial rate of $D H$ change. The exponential term of the right side describes the decreasing $D H$ rate. Therefore, the physical meaning of $\mathrm{b}$ can be understood as the $D H$ rate relaxation constant, which describes how fast the enzyme is

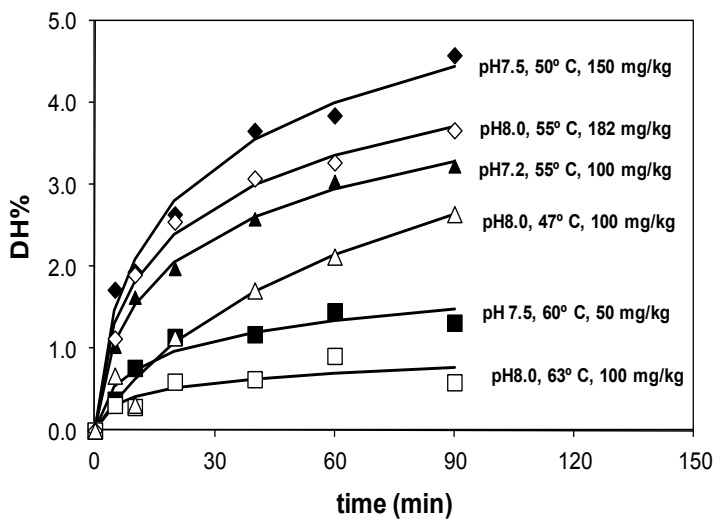

Figure 2: Fitting kinetics curves to GonzalezTello's model (Gonzalez-Tello et al., 1994) through equation 11. Hydrolyzing conditions are specified on the curves. Points are experimental data and the lines are plotted by fitted value.

denatured. Initial rates of $\% \mathrm{DH}$, i.e. a value, are summarized in figure 3. Apparently, the a values can be divided into two groups. Within each group, a values increase with an increase in enzymatic dose, suggesting that enzyme dose may be the primary factor determining the initial hydrolysis rate. Generally, the a values in the lower group are from hydrolysis at either high $\mathrm{pH}$ or high temperature. Thus, these conditions are not favorable for hydrolysis.

$D H$ rate relaxation constants, i.e. $b$ values, were pooled in figure 4. Apparently, $b$ values were strongly correlated to temperature, suggesting that temperature may be the primary factor determining $b$ values. Higher temperature resulted in larger $b$ value, implying higher denaturing rate in the experimental temperature range. The $\mathrm{b}$ values for hydrolysis at $55^{\circ} \mathrm{C}$ were plotted against enzyme dose in figure 5 . Interestingly, the $b$ values are higher at lower enzyme dose, while $\mathrm{pH}$ did not show much effect on $b$ values.

A hydrolysis favorable for industrial manufacture is one with a higher initial rate ( $a$ value) and smaller rate relaxation constant ( $b$ value). From the above analysis (see figures 3,4 and 5), a higher dose resulted in larger $a$ and smaller $b$ values, therefore, it is always favorable for the 


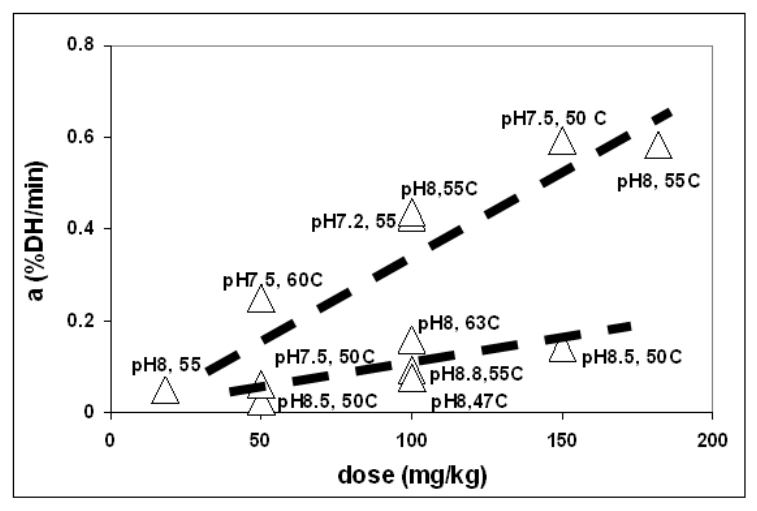

Figure 3: Initial rate ( $a$ value) versus enzyme dose for hydrolysis at different $\mathrm{pH}$ and temperature.

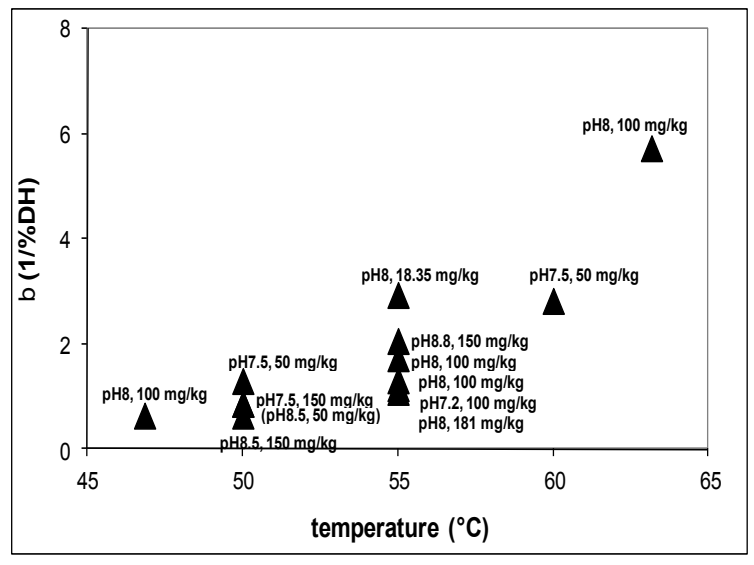

Figure 4: Rate relaxation constant (b value) versus temperature for hydrolysis at different $\mathrm{pH}$ and enzyme dose.

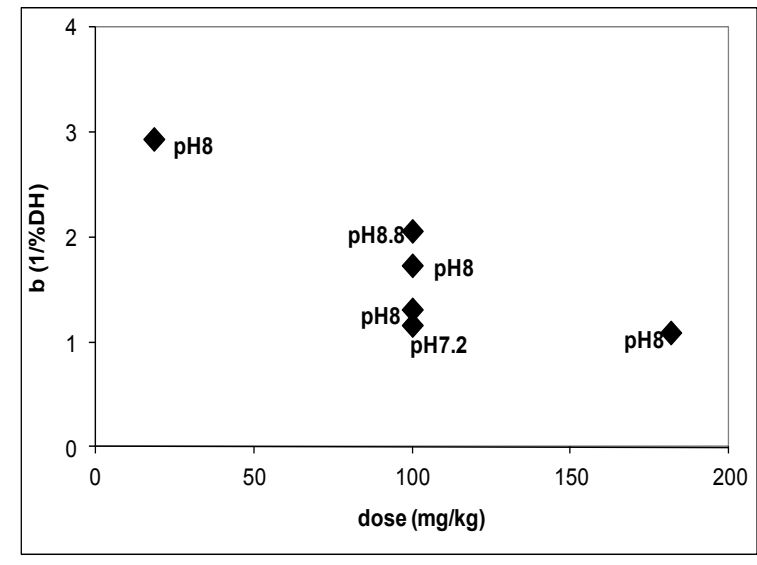

Figure 5: ( $b$ value) versus enzyme dose for hydrolysis at $55^{\circ} \mathrm{C}$ and different $\mathrm{pH}$.

hydrolysis. On the other hand, higher temperature resulted in larger $b$ values, but may also result in lower a values. Therefore, an optimal temperature exists for the hydrolysis. These conclusions are consistent with common practice in enzymatic reaction, which may suggest that the model is reasonable.

\section{A conceptual simulation example}

Enzymatic hydrolysis reactions are usually sensitive to $p H$, temperature, enzyme dose and substrate concentration. When a hydrolysis reaction is conducted at the bench, these factors are able to be controlled fairly accurately and consistently. However, when such reactions are conducted in a manufacturing facility, distribution of and variation in these factors are usually not negligible. These distributions and variations contribute to the variability of the final product. In a reaction tank, the $p H$, temperature, enzyme dose and substrate concentration are not always homogeneous. Consequently, the hydrolysis at different locations in the tank may occur under different conditions. Therefore, the rate of hydrolysis may be different at different locations in the tank as well as time of reaction.

Theoretically, the product is produced as:

$$
\frac{d P}{d t}=f(x, y, z, t)
$$


Hydrolysis Kinetics of Soy Protein and its Application |31

And the final product is:

$$
P=\int_{V} \int_{t}\left(\frac{d P}{d t}\right) d V d t
$$

For a process simulation, directly obtaining $f(x, y, z, t)$ is not practical. A practical approach is to determine $\frac{d P}{d t}$ as a function of $\mathrm{pH}$, temperature, enzyme dose and substrate, which can be obtained through bench experiments under well controlled conditions. Then distributions of these variables are calculated by computational fluid dynamics software (Xia \& Sun, 2002). The combination of the two makes it feasible to simulate the hydrolysis kinetics in the tank. To demonstrate such a concept, a simple simulation is demonstrated below: A hydrolysis was conducted in a tank at $\mathrm{pH} 8.0$ with enzyme dose of $100 \mathrm{mg} / \mathrm{kg}$ protein, at protein concentration of $14 \%(\mathrm{w}: \mathrm{w})$. To simplify the simulation, $\mathrm{pH}$, enzyme and substrate are assumed to be homogeneous in the tank due to good mixing but the heating system is not stable with random variation. The $a$ and $b$ values at $46.8,55$ and $63.2{ }^{\circ} \mathrm{C}$ under various enzyme dose and $\mathrm{pH}$ are listed in table 3.2. At any time point, the temperature is random within an operating limit. The DH rate, $\frac{d D H}{d t}$, at any temperature could be obtained by interpolating between $D H$ rates at known temperatures. For example, at $\% \mathrm{DH}$ of $0.37 \%, \frac{d P}{d t}$ at $46.8{ }^{\circ} \mathrm{C}$ can be calculated to be 0.060468 using $a$ and $b$ values of 0.07663 and 0.6407 , respectively. Similarly, $\frac{d D H}{d t}$ at $55^{\circ} \mathrm{C}$ at the same $\% \mathrm{DH}$ can be calculated to be 0.269419 using $a$ and $b$ values of 0.4378 and 1.3132 , respectively. At a temperature of $52{ }^{\circ} \mathrm{C}, \frac{d D H}{d t}$ can be obtained by interpolating the two data points, which gives a result of 0.192646 . Using this approach, equation 10 is integrated numerically for random temperature profiles to give $\% \mathrm{DH}$ at any time. Figure 6 shows the simulation results. Under ideal control, the temperature is consistently $55{ }^{\circ} \mathrm{C}$, resulting in a $\% \mathrm{DH}$ profile shown as curve 1 . In reality, the temperature is oscillating within a control limit. For example, if the control limit is $52-57^{\circ} \mathrm{C}$, a temperature profile may be a random curve (e.g. curve B), resulting in the $\% \mathrm{DH}$ curve 2. Similarly, if the temperature control is poor, the temperature profile may be within $50-60{ }^{\circ} \mathrm{C}$ (curve $\mathrm{C}$ or $\mathrm{D}$ ). By conducting the simulation for a large number of times, variation of the final

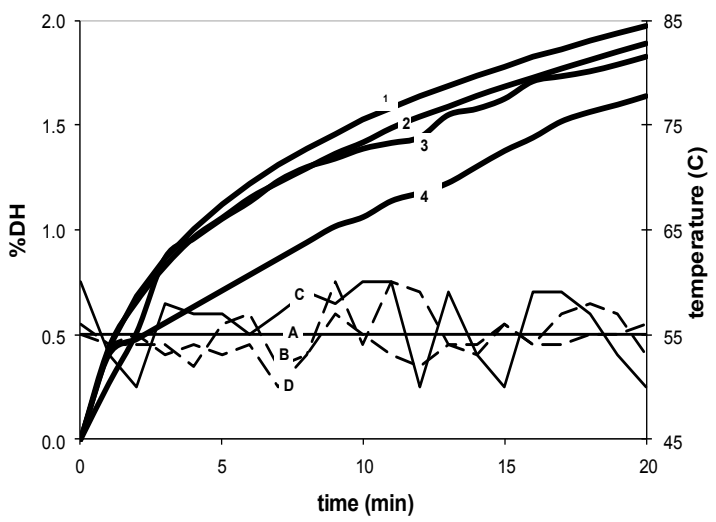

Figure 6: Simulation of hydrolysis under temperature variation at $\mathrm{pH} 8$ and enzyme dose of 100 $\mathrm{mg} / \mathrm{kg}$ protein. The temperature profiles were created using random number generator in Excel. Curve A: ideal temperature of $55{ }^{\circ} \mathrm{C}$; Curve B: random temperature within $52-57^{\circ} \mathrm{C}$; Curve $\mathrm{C} \&$ D: random temperature within $50-60{ }^{\circ} \mathrm{C}$. Curves 1-4 are simulated $\% \mathrm{DH}$ curves corresponding to temperature profiles A-D respectively.

products may be estimated. In this simple simulation, the temperature range of $52-57{ }^{\circ} \mathrm{C}$ gave fairly small variation (data not shown), while a temperature range of $50-60{ }^{\circ} \mathrm{C}$ resulted in rather large variation, as shown by $\% \mathrm{DH}$ curves 3 and 4.

\section{Conclusion}

Due to the reaction complexity, process control is more difficult for enzymatic hydrolysis than usual chemical reactions. Mathematical simulation is a valuable tool to understand effect and sensitivity of reaction unit geometry and operating parameters. The validity of a simulation depends on many factors, among which reaction kinetics at different conditions are critical. Due to the complexity of the substrate, the Michaelis-Menten kinetics model is usually not recommended for a food protein hydrolysis reaction. In the current work, we showed that the Gonzalez-Tello kinetics model described the experimental data fairly well and could be conveniently applied in simulation. A simple, concep-

\begin{tabular}{l|l|l|l} 
IJFS & April 2012 & Volume 1 & pages 26-32
\end{tabular} 
tual simulation was conducted to demonstrate the approach and value of such a simulation. Applying such an approach with computational fluid dynamics software makes it possible to provide valuable guidelines for scaling up a process and setting control limits.

\section{Acknowledgements}

The authors thank Pat Maher and Myron Lewis for their support in degree of hydrolysis analysis.

\section{References}

Barros, R., \& Malcata, F. (2002). Modeling the kinetics of whey protein hydrolysis brought about by enzymes from cynara cardunculus. Journal of Agricultural and Food Chemistry, 50(15), 4347-4356. doi:10. 1021/jf0200319. eprint: http://pubs.acs . org/doi/pdf/10.1021/jf0200319

Ely, R., Williamson, K. J., Guenther, R. B., Hyman, M., \& Arp, D. (1995). A cometabilic kinetics model incorporating enzyme inhbition, inactivation, and recovery: i. model development, analysis, and testing. Biotechnology and Bioengineering, 46(3), 218-231. doi:10.1002/bit.260460305

Erdmann, K., Cheung, B. W., \& Schröder, H. (2008). The possible roles of food-derived bioactive peptides in reducing the risk of cardiovascular disease. The Journal of $\mathrm{Nu}$ tritional Biochemistry, 19(10), $643-654$. doi:10.1016/j.jnutbio.2007.11.010

Food and Drug Administration. (1999). Food labeling: health claims; soy protein and coronary heart disease. 64 fed. reg. 57699. USDA.

Gibbs, B., Zougman, A., Masse, R., \& Mulligan, A. (2004). Production and characterization of bioactive peptides from soy hydrolysate and soy-fermented food. Food Research International, 37(2), 123 -131. doi:10.1016/ j.foodres.2003.09.010

Gonzalez-Tello, P., Camacho, F., Jurado, E., Paez, M. P., \& Guadix, E. M. (1994). Enzymatic hydrolysis of whey proteins: i. kinetic models. Biotechnology and Bioengineering, 44(4), 523-528. doi:10.1002/bit.260440415
Markovic, I., Topolovec, V., Maric, V., \& Johanides, V. (1988). The barley protein degradation: mechanism of protein solubilization during barley mashing with neutral proteinase. Biotechnology and Bioengineering, 32(1), 18-27. doi:10. 1002 / bit . 260320105

O’Meara, G., \& Munro, P. (1985). Kinetics of the hydrolysis of lean meat protein by alcalase: derivation of two alternative rate equations and their fit to experimental data. Biotechnology and Bioengineering, 27(6), 861-869. doi:10.1002/bit.260270616

Pusky, G. (1975). Modification of functional properties of soy proteins by proteolytic enzyme treatment. Cereal Chemistry, 52(5), 655664. Retrieved from http : / / www . aaccnet . org / cerealchemistry / backissues / 1975/chem52_655.pdf

Were, L., Hettiarachchy, N., \& Kalapathy, U. (1997). Modified soy proteins with improved foaming and water hydration properties. Journal of Food Science, 62(4), 821824. doi:10 . $1111 /$ j . 1365 - 2621 . 1997. tb15463.x

Wong, T., Kerr, P., Ghosh, P., Lombardi, J., Maldonado, Y., Lynglev, G., ... Oestergaard, P. (2008). U.S. Patent No. 305,212. Washington, DC: U.S. Patent and Trademark Office.

Xia, B., \& Sun, D.-W. (2002). Applications of computational fluid dynamics (cfd) in the food industry: a review. Computers and Electronics in Agriculture, 34(1-3), 5-24. doi:10.1016/S0168-1699(01)00177-6 